\title{
Experimental Status of Proton Decay
}

\author{
J. C. VAN DER VELDE \\ Physics Department \\ University of Michigan \\ Ann Arbor, Michigan 48109
}

Question: How can we even be here?

Answer: Baryon number is not conserved.

This is a seemingly paradoxical answer to the long-standing mystery of why there appears to be a preponderance of matter over antimatter in the universe. The basic interaction that produced baryons in the early instants after the "big bang" did not conserve baryon number. Therefore, when the last baryon-antibaryon annihilations occurred, "we" were left with an excess of baryons.

As I write this, I look out over the mountains of Aspen. There is a lot of baryon number sitting there. Do the anti-mountains of anti-Aspen exist somewhere? Apparently not.

There are other theoretical reasons for believing that the quarks inside baryons should be able to change directly into leptons via a new interaction and leave behind no baryon number. Hence, it would be nice if the hypothesis of baryon number nonconservation could be confirmed and quantified by direct measurement. This is what has motivated the experimenters to delve into that which I will describe here.

We begin with a list of six current experiments having data on nucleon decay, which is shown in TABLE 1. The first column gives the fiducial mass from those candidate events that are accepted. The first three experiments use tracking calorimeters in which the principal mass is in the form of iron plates. The last three are of the water Cherenkov type. The analyzed exposure and number of events found within the fiducial volume are given for each experiment in the last two columns. A more detailed description of these experiments can be found in the recent review by Perkins. ${ }^{1}$

Contained event rates per kilotonne-yr of exposure are shown in FIGURE 1. One sees that the rates are generally in good agreement at a value of $\sim 100$ events/kt-yr. This is approximately the rate expected ${ }^{2,4}$ due to atmospheric neutrino interactions above a threshold neutrino energy of about $300 \mathrm{MeV}$. The Kamioka experiment has a somewhat lower threshold than the others and sees a correspondingly higher rate. The rate at KGF is expected to be a little lower because of their lower geomagnetic latitude. The HPW group has only searched for events with $\geq 2$ muon decays and therefore sees a much smaller rate.

The rates shown in FIGURE 1 have not been corrected for detection efficiency. Typical efficiencies for nucleon decays and neutrino interactions are in the range of 50-90\%.

The arrows at $6(60)$ events/kt-yr represent the rate of events expected if the nucleon lifetime were $10^{32}\left(10^{31}\right) \mathrm{yr}$, assuming $100 \%$ detection efficiencies and equal rates for protons and neutrons. From this, one sees immediately that one is faced with a formidable neutrino background problem in searching for nucleon decays at a lifetime level of $10^{32}$ years or longer. 
TABLE 1. Current Proton Decay Experiments ${ }^{a}$

\begin{tabular}{lccrc}
\hline \multicolumn{1}{c}{ Experiment } & $\begin{array}{c}\text { Fiducial } \\
\text { Mass }\end{array}$ & $\begin{array}{c}\text { Magnetic } \\
\text { Latitude }\end{array}$ & $\begin{array}{c}\text { Analyzed } \\
\text { Exposure }\end{array}$ & $\begin{array}{c}\text { Contained } \\
\text { Events }\end{array}$ \\
\hline Kolar G.F. & 70 tonne $\mathrm{Fe}$ & $2^{\circ} \mathrm{N}$ & 210 tonne-yrs & 16 \\
NUSEX & 100 tonne $\mathrm{Fe}$ & $50^{\circ} \mathrm{N}$ & 220 tonne-yrs & 22 \\
FRÉJUS & 450 tonne $\mathrm{Fe}$ & $50^{\circ} \mathrm{N}$ & 90 tonne-yrs & 9 \\
IMB & 3300 tonne $\mathrm{H}_{2} \mathrm{O}$ & $52^{\circ} \mathrm{N}$ & 3800 tonne-yrs & 401 \\
KAMIOKANDE & 880 tonne $\mathrm{H}_{2} \mathrm{O}$ & $27^{\circ} \mathrm{N}$ & 660 tonne-yrs & 89 \\
HPW & 560 tonne $\mathrm{H}_{2} \mathrm{O}$ & $50^{\circ} \mathrm{N}$ & 380 tonne-yrs & $5^{b}$ \\
\hline
\end{tabular}

TABLE 1 is an updated version of a similar one recently presented by LOSECCO, J. 1984. Invited paper at the APS-DPF annual meeting, Santa Fe, New Mexico.

$2 \mu+3 \mu$ only.

The small circles represent the rate at which the various experiments find events that are consistent with one or more nucleon decay modes. These events are usually referred to as "candidates." Given an event with more than just a single track, a detector with a less-than-ideal resolution, and some amount of imagination, one can usually find some nucleon decay mode that fits it. Hence, one should be somewhat wary about the term "candidate" at this point. So far, the candidates do not show the clustering about a dominant decay mode that one would expect for real nucleon decays.

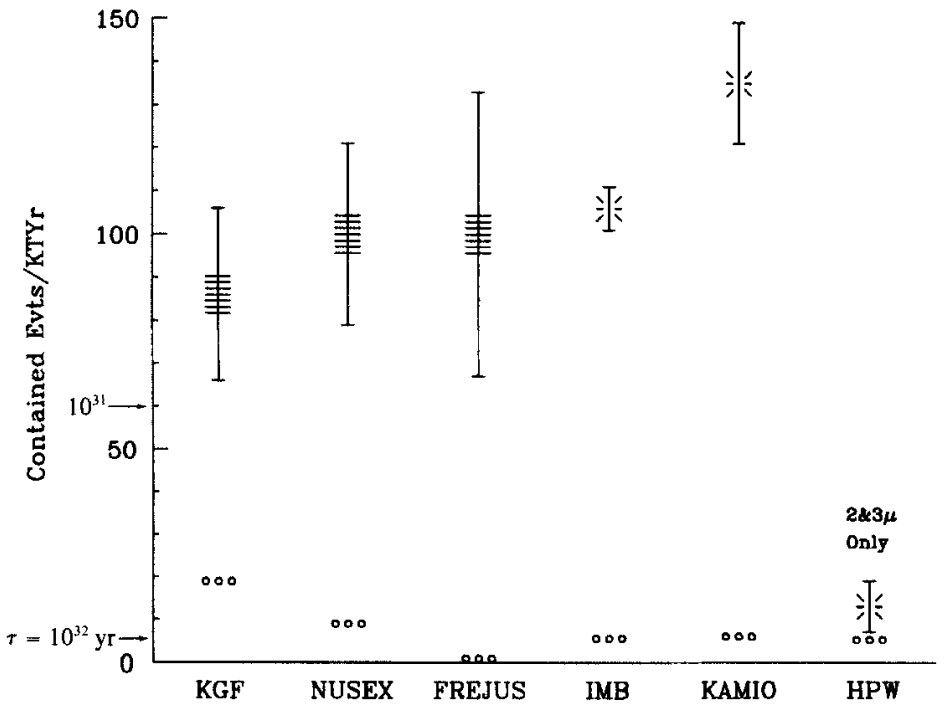

FIGURE 1. Contained event rates for six current nucleon decay detectors. The first three points are from iron-plate tracking devices and the second three are from water Cherenkov detectors. The overall rates (top points with error bars) are in reasonable agreement with each other and with the expectations based on atmospheric neutrino fluxes. Arrows at the left represent the total expected event rate from the decay of protons and neutrons (assuming they had the same lifetime). The small circles represent reported rates of "candidate" events for nucleon decay. (See text.) 
In setting lower limits on lifetime/branching ratios $(\tau / B)$ for various decay modes, most experimenters have taken the conservative attitude that all of the candidates for that particular mode are real. Background estimates for various modes are still fairly crude, so that, generally, background has not been subtracted from the candidate samples in calculating limits. Quoted lower limits on $\tau / B$ are, therefore, generally quite conservative.

As can be seen from FIGURE 1, candidates are being found at a level of $5 \%$ to $10 \%$ of the contained events. This is just about the rate that atmospheric neutrino interactions start to mimic nucleon decays. We illustrate the problem in Figure 2. A proton with Fermi momentum can decay into $\mu^{+} \pi^{0}$ with an included angle of $150^{\circ}$ or more. This configuration can be mimicked by the reaction,

$$
\nu_{e}+\mathrm{n} \rightarrow \mathrm{n}+e^{-}+\pi^{+},
$$

where the $e^{-}$makes a shower and the $\pi^{+}$decays into a $\mu^{+}$. Making a cut of $\theta>150^{\circ}$ on

FIGURE 2. A typical low energy single pion production reaction by an atmospheric electron neutrino. Such wide-angle two-track events are a serious source of background in (earthbound) nucleon decay detectors if the nucleon lifetime is $>10^{32}$ yrs.

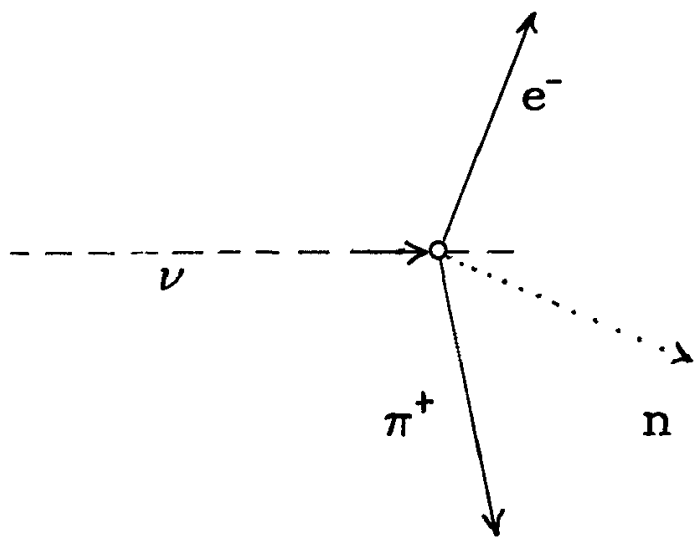

such two-track events saves $\sim 90 \%$ of the decays from a nucleus like oxygen and rejects a large fraction, but not all, of the neutrino background around $1 \mathrm{GeV}$. (We will give a more precise estimate later in the paper.)

of course, a wide-angle two-track event can be mimicked by a single track that scatters through a small angle if one does not know the track directions. Hence, given the present limits on the nucleon lifetime and the present knowledge of neutrino background, it is essential to be able to measure track directionality. This is straightforward for the water Cherenkov detectors, but more difficult for tracking calorimeters since they must rely on ionization measurements, shower profiles, multiple scattering, etc.

Understanding the properties of neutrino background has become the watchword for nucleon decay experimenters. It will also be the watchword for the remainder of this paper.

We now give a brief review of the present results from the six experiments listed. At the end we will say something about experiments planned for the near future. 


\section{KGF RESULTS}

Collaborating Institutions:

Tata Institute of Fundamental Research

Osaka City University

Detector Characteristics:

Fiducial mass $=70$ tonnes

$10 \times 10 \mathrm{~cm}$ proportional tubes with $0.23 \mathrm{~cm}$ iron walls

$1.2 \mathrm{~cm}$ iron plates

Depth $=7600$ mwe

Ionization measurement gives some track directionality

Results:

210 tonne-yr exposure

16 contained events

4 nucleon decay candidates

The KGF detector is comprised of two crossed horizontal stacks of large ironwalled proportional tubes, $6 \mathrm{~m} \times 4 \mathrm{~m}$ in one view and $4 \mathrm{~m} \times 4 \mathrm{~m}$ in the other. The

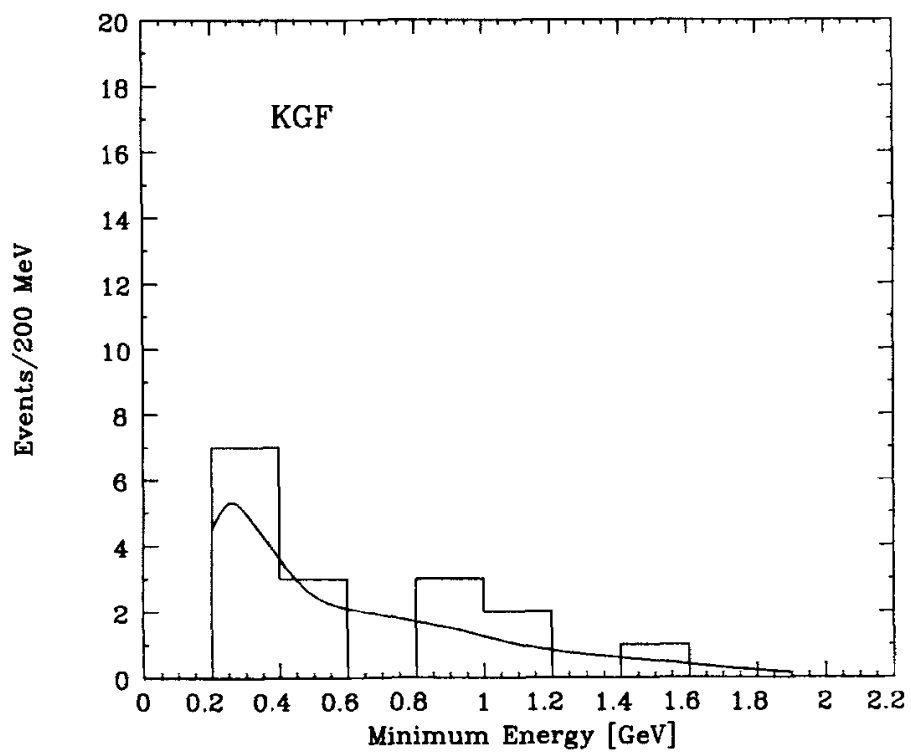

FIGURE 3. Energy distribution of KGF contained events. The curve is the KGF estimate of neutrino background. 


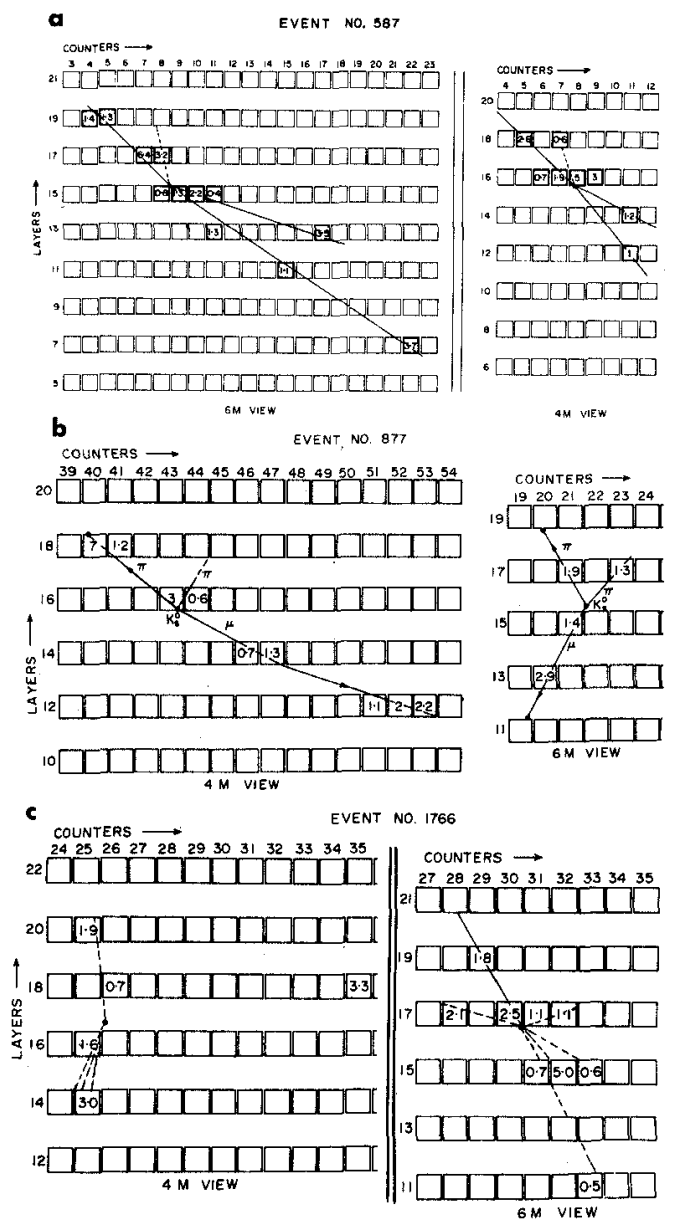

FIGURE 4. (a) KGF candidate for $P \rightarrow e^{+} \pi^{\circ}$. Widely spaced hits indicate an electromagnetic shower. (b) KGF candidate for $P \rightarrow \mu^{+}\left(K^{\circ} \rightarrow \pi^{+} \pi^{-}\right)$. Numbers in boxes indicate the ionization level in the associated proportional tube. (c) KGF candidate for $N \rightarrow \nu \eta^{\circ}, N \rightarrow e^{+} \rho^{-}$, or $P \rightarrow$ $e^{+} \pi^{\circ}$.

experiment was the first dedicated nucleon decay apparatus to turn on, in recent times, and has now logged more than three years of livetime. The visible energy spectrum of 16 contained events is shown in FIGURE 3. The estimated background due to neutrino interactions is also shown, along with the position of four nucleon decay candidates. Orthogonal views of three of the candidates are shown in FiguRES 4a, b, and c. The ionization level recorded by each tube is also indicated. The experimenters make use of this information to get track directionality. Corrections for the amount of gas traversed by a hypothesized track can be a significant factor in the ionization information.

As can be seen from FIGURE 4, the spatial resolution and number of tubes firing in 
this pioneering experiment appear to be minimal, which is due to the tube size and amount of iron between tubes. One would hope to have more information content per event in order to make a convincing case for the discovery of nucleon decay in the midst of the neutrino background, especially given what is now known about the rate.

\section{NUSEX RESULTS}

Collaborating Institutions:

Frascati, Milano, Torino, CERN

Detector Characteristics:

Fiducial mass $=100$ tonnes

$9 \mathrm{~mm} \times 9 \mathrm{~mm}$ limited streamer tubes, bidirectional readout

$1.0 \mathrm{~cm}$ iron plates

No ionization information

Depth $=5000$ mwe

Results:

220 tonne-yr exposure

22 contained events

2 nucleon decay candidates

This detector has better spatial resolution than that of KGF due to the smaller tube size and thinner iron plates. The two nucleon decay candidates are shown in FIGURES $5 \mathrm{a}$ and $\mathrm{b}$.

The NUSEX experimenters determine the neutrino background from the results of running a test module of the detector in a neutrino beam at CERN. In principle, such tests can be made quite realistic for tracking calorimeter detectors because of their modular nature. In practice, difficulties arise in trying to match the atmospheric neutrino spectrum and $\nu_{e}, \bar{\nu}_{e}, \nu_{\mu}, \bar{\nu}_{\mu}$ content with an accelerator beam. Also since tracking detectors do not have isotropic response, it is difficult to obtain sufficient statistics at a sufficiently large number of incident angles.

The NUSEX group calculates the background for event \#1 to be $0.07 \pm 0.05$ events. They have also put a test module into a gamma beam at an incident angle of $45^{\circ}$ to help them calculate the background for event \#2 to be $0.18 \pm 0.06$ events.

\section{FRÉJUS RESULTS}

Collaborating Institutions:

Aachen, Orsay, Palaiseau, Saclay, Wuppertal 

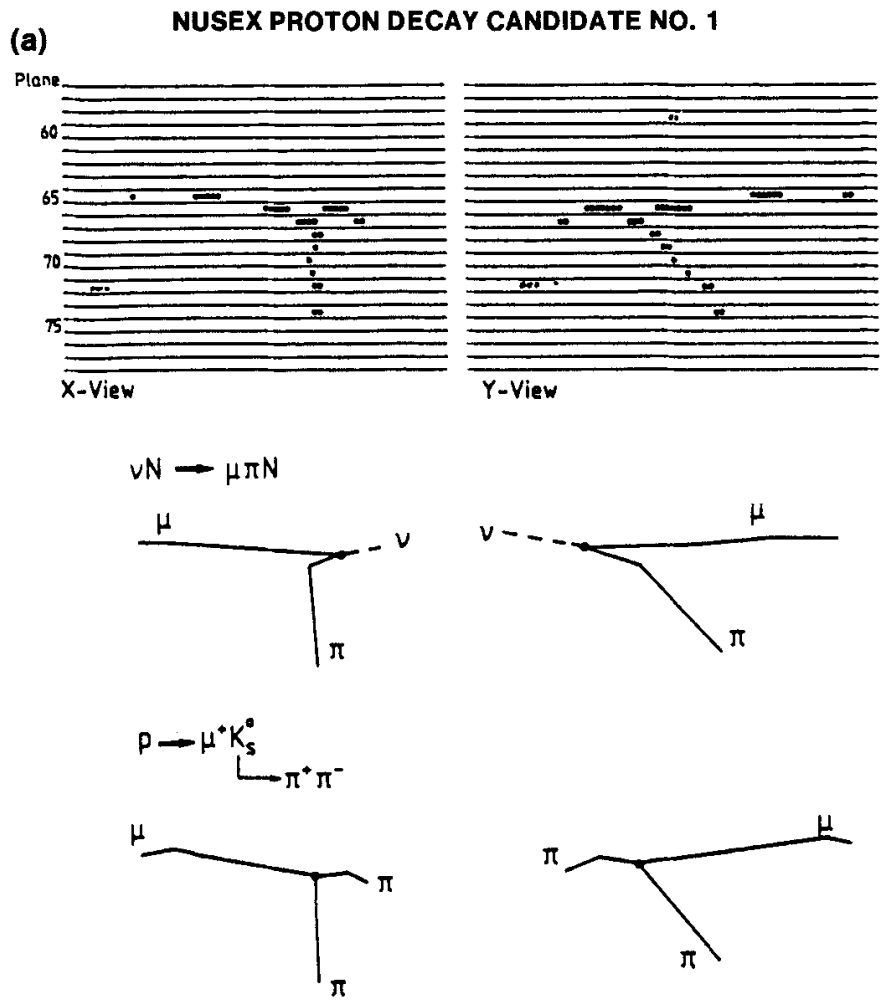

(b)
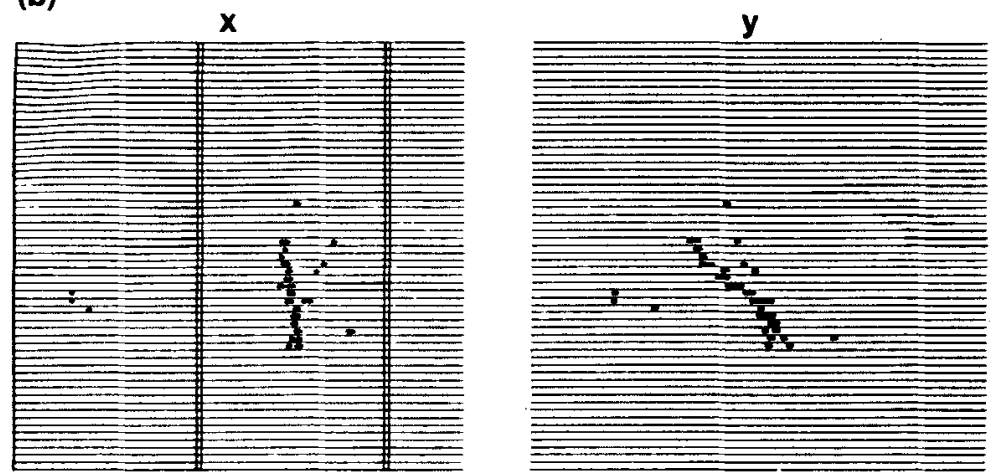

\section{Event 2}

FIGURE 5. (a) NUSEX candidate for $P \rightarrow \mu^{+}\left(K^{\circ} \rightarrow \pi^{+} \pi^{-}\right)$. This interpretation and the interpretations as a single pion production by a neutrino interaction are depicted below the hit patterns in the two views. Assuming the decay hypothesis, the total energy in the event is $1.0 \pm 0.2$ $\mathrm{GeV}$, the invariant mass of $K^{\circ}$ is $550 \pm 80 \mathrm{MeV}$, and the momentum imbalance is $\Delta P=0.4 \pm 0.2$ $\mathrm{GeV} / c$. (b) NUSEX candidate for $P \rightarrow e^{+} \pi^{\circ}$. Assuming two showering tracks, the total energy is $1.1 \pm 0.2 \mathrm{GeV}$ and $\Delta P=0.25 \pm 0.15 \mathrm{GeV} / c$. 
Detector Characteristics:

Fiducial mass $=450$ tonnes

$6 \mathrm{~mm} \times 6 \mathrm{~mm}$ flash tubes triggered by $15 \mathrm{~mm} \times 15 \mathrm{~mm}$ Geiger tubes

$3 \mathrm{~mm}$ thick iron plates

No ionization information

Depth $=4200$ mwe

Results:

90 tonne-yr exposure

9 contained events

0 nucleon decay candidates

This detector has excellent spatial resolution because of the thin plates and small flash tubes. It can also detect muon decays with an efficiency of $\sim 70 \%$. Early results give them nine contained events at the rate expected for neutrino interactions. None of the events appears to be of an unusual variety. Figure 6 shows an $800 \mathrm{MeV}$ muon that starts and stops inside the detector. The good spatial resolution allows one to see the multiple scattering of tracks like this.

\section{HPW RESULTS}

\section{Collaborating Institutions:}

Harvard, Purdue, Wisconsin

Detector Characteristics:

Fiducial mass $=560$ tonnes

Water Cherenkov type

7005 -inch phototubes distributed in cylindrical volume

Walls lined with mirrors for increased light collection

PWC anti surrounds tank

Depth $=1500 \mathrm{mwe}$

Results:

380 tonne-yr exposure

5 contained events ( $\geq 2$ muon decays only)

2 nucleon decay candidates

Until now, the experimenters have only used their data to look for events with two or more muon decays. This, along with their system of PWC tubes, more easily allows them to filter out cosmic ray muon background. They make a further cut at 300 photoelectrons, which corresponds roughly to accepting events with Cherenkov equiva- 
lent energy $\left(E_{c}\right) \leq 450 \mathrm{MeV}$. The $2-\mu$ sample so selected is shown in Figure $7 .^{3}$ The sample is further reduced to four contained events by scanning away entering showers and electronic noise, as shown in FIGURE 7. A similar procedure is applied to the 3- $\mu$ sample, which leaves them with one contained event having 92 MTD hits $(\approx$ photoelectrons) in the primary vertex trigger.

Separate vertices for the primary trigger and muon decay positions are determined with an accuracy of $\approx \pm 1 \mathrm{~m}$ for each event. These data are shown in TABLE 2 . Events 2 and 3 are chosen as possible nucleon decay candidates because their "Isotropy" variable makes them look more isotropic (proton-decay-like) than the general neutrino background events. The vertex locations for the two candidates are depicted in FIGURES $8 \mathrm{a}$ and $\mathrm{b}$.

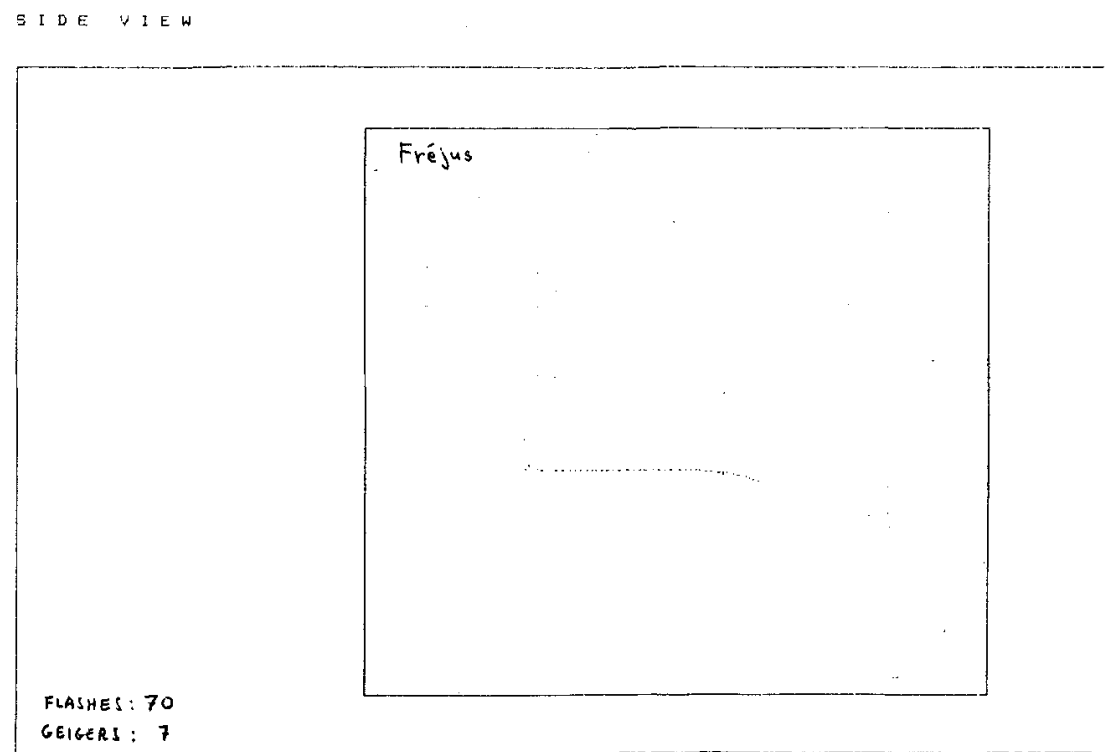

FIGURE 6. Event in the new Fréjus detector. An $0.8 \mathrm{GeV}$ horizontal muon, probably made by a neutrino interaction, starts and stops deep inside the detector volume.

The experimenters calculate the total neutrino background for the five events to be $\sim 0.3$ events. This calculation is in reasonable agreement with IMB results of five events with $\geq 2$ muons having $E_{c}<450 \mathrm{MeV}$ since the IMB data are based on ten times the exposure.

It seems clear that the five HPW contained events cannot be due to neutrino interactions. The experimenters, though, suggest that they may be interesting: perhaps multimuon/pion modes of nucleon decay.

There seems to be a problem with all five of the events in that the number of MTD hits associated with the primary vertex (trigger 1 ) does not indicate enough Cherenkov energy $\left[E_{c} \approx 1.5\right.$ (MTD hits)]. That is, one expects factors of three to ten times more 


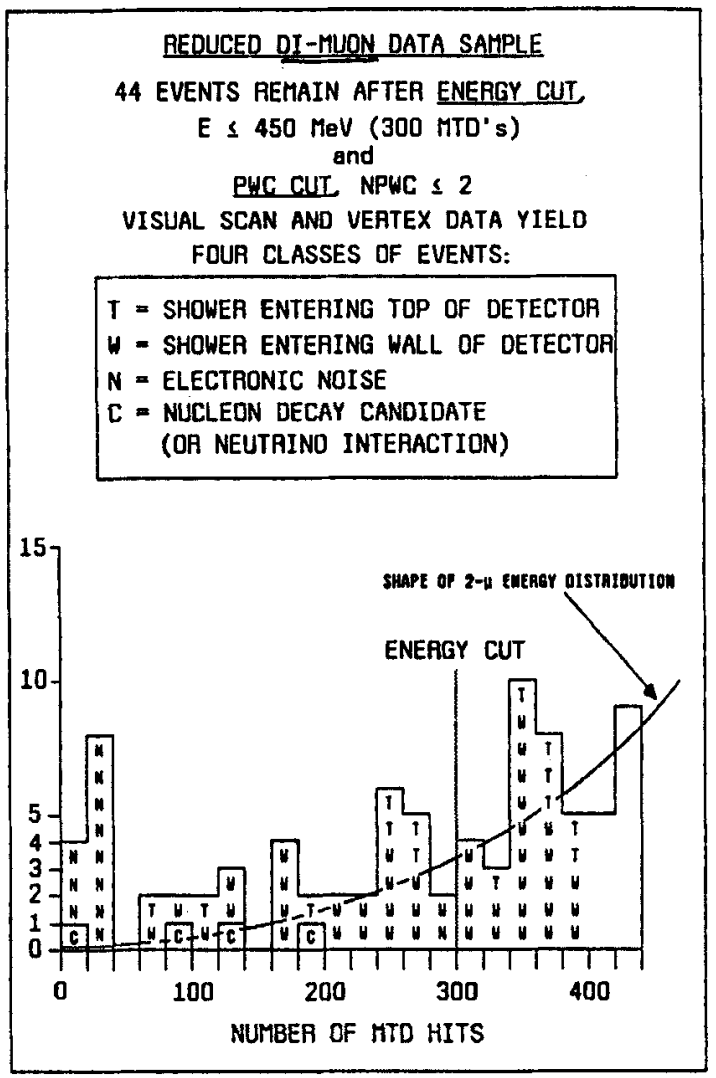

FIGURE 7. HPW sample of events with two muon decays. The energy cut ( 300 MTD hits) is indicated, along with the scanning results on each event.

TABLE 2. HPW Contained Events

\begin{tabular}{lccccc}
\hline & Trig. $^{a}$ & MTDs $^{b}$ & Iso. $^{c}$ & Vertex $(x, y, z)$ & $\begin{array}{c}\mu \text {-Decay } \\
\text { Time }(\mu)\end{array}$ \\
\hline Event 1 & 1 & 162 & .34 & $-1.6,2.9,1.3$ & \\
{$[1429-11,137]$} & 2 & 22 & & $-1.5,-1.5,1.5$ & 0.6 \\
& 3 & 39 & & $-1.5,2.6,1.6$ & 1.5 \\
Event 2 & 1 & 17 & .36 & $3.2,-1.7,0.2$ & \\
{$[2122-5236]$} & 2 & 42 & & $3.3,-2.1,-2.0$ & 1.2 \\
& 3 & 38 & & $1.1,-3.5,-1.5$ & 2.6 \\
Event 3 & 1 & 81 & .21 & $3.1,-2.3,-0.1$ & \\
{$[2133-16,773]$} & 2 & 32 & & $3.5,-1.0,2.5$ & 0.9 \\
& 3 & 25 & & $1.5,-2.5,2.5$ & 2.4 \\
Event 4 & 1 & 132 & .48 & $2.3,-2.0,1.6$ & \\
{$[2489-2499]$} & 2 & 14 & & $3.0,-2.0,0.0$ & 0.9 \\
& 3 & 33 & & $2.4,0.3,2.3$ & 1.9 \\
Event 5 & 1 & 92 & .40 & $2.8,-2.5,2.3$ & \\
{$[2920-32,257]$} & 2 & 24 & & $3.5,-3.5,2.5$ & 2.4 \\
& 3 & 40 & & $2.0,-2.0,2.5$ & 3.2 \\
1 PWC HIT & 4 & 15 & & $2.5,0.5,3.5$ & 4.3 \\
\hline
\end{tabular}

\footnotetext{
Trigger number.

"Number of MTD hits.

'Isotropy.
} 
light to be recorded if muons or other charged particles are to propagate from the primary vertex to the decay vertices. The experimenters attribute this inconsistency to possible errors in the individual vertex location estimates, i.e., the vertices are really much closer together than indicated by the positions given in TABLE $2 .^{3}$

One can ask if there is any evidence for HPW type events in the other detectors. (Only the water Cherenkov experiments so far have low enough thresholds and high enough muon detection efficiencies to see such events.) If one takes the number of MTD hits in TABLE 2 at face value, then three of the five events are below the present

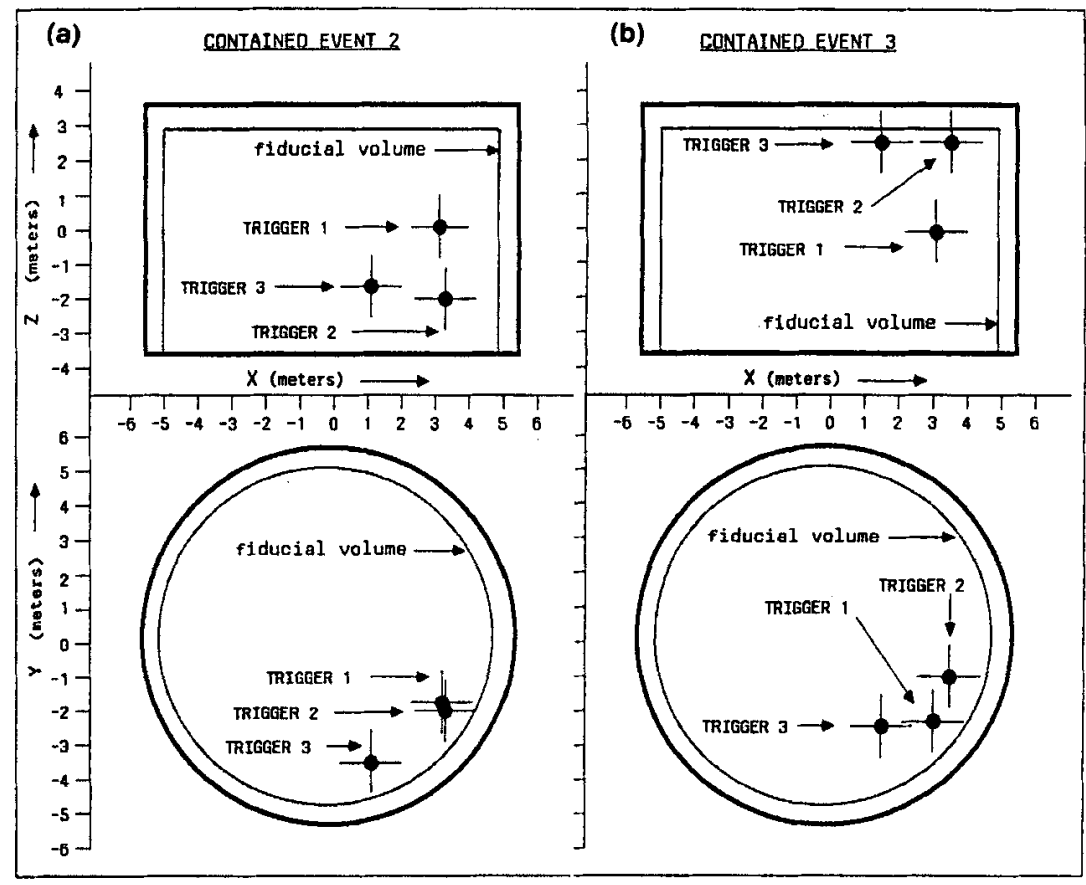

EVENT 2 VERTICES

EVENT 3 VERTICES

FIGURE 8. (a, b) HPW event candidates for nucleon decay. Projections of the event vertices are shown in top and side views. Trigger 1 is the primary vertex and triggers 2 and 3 are the positions of the two muon decays. More details for these and three other contained events can be found in TABLE 2.

IMB threshold of $E_{c}=180 \mathrm{MeV}(\approx 120 \mathrm{MTD}$ hits in HPW $)$. With ten times the HPW exposure, IMB sees ten contained events with $2-\mu$ decays and none with 3- $\mu$ decays. Only one of these events has an "Isotropy" of $\leq 0.5$. It has $E_{c}=350 \mathrm{MeV}$. We will take a closer look at it later (in FIGURE 15a). Hence, whereas IMB might have expected to see $\sim 20 \pm 14 \mathrm{HPW}$ type events, only one is seen.

In principle, the KAMIOKANDE experiment can detect events with several muon decays at $E_{c}$ values of $\leq 50 \mathrm{MeV}$. Hence, it should be able to check the HPW results on a possible multimuon signal concentrated below $E_{c}=100 \mathrm{MeV}$. At present, I don't 
have the appropriate information from KAMIOKANDE in order to make the comparison. KAMIOKANDE does, however, have almost twice the exposure of HPW.

\section{KAMIOKANDE RESULTS}

\section{Collaborating Institutions:}

University of Tokyo (Department of Physics, International Center for Elementary Particle Physics, and Institute for Cosmic Ray Research), National Lab for High Energy Physics (KEK), and University of Niigata

\section{Detector Characteristics:}

Fiducial mass $=880$ tonnes

Water Cherenkov type

100020 -inch phototubes on surface of cylinder

$20 \%$ area coverage

Depth $=2700 \mathrm{mwe}$

Results:

660 tonne-yr exposure

89 contained events

4 nucleon decay candidates

Preliminary results on the energy spectrum of the KAMIOKANDE events are shown in Figure 9. Only 65 of the 89 events, those that appear to be just a single track (pseudo-elastic), are shown. The energy spectrum agrees, at least approximately, with the expectation from atmospheric neutrinos, although the atmospheric predictions for the total rate and $\nu_{u} / \nu_{e}$ ratio are not very well determined below $500 \mathrm{MeV}^{4}$ It is significant that KAMIOKANDE sees events with less than $100 \mathrm{MeV}$ of Cherenkov energy. This is an area totally unexplored by any other current (or previous) detector.

In addition to the 65 pseudo-elastic events in FIGURE 9, the KAMIOKANDE group has 24 contained events with $\geq$ two tracks. If the tracks (i.e., Cherenkov cones) are well isolated from one another, the distinction between showering $(S)$ or nonshowering $(M)$ tracks can be made. The group lists four candidates consistent with one or more nucleon decay modes. ${ }^{5}$

The first of these is the rather unusual event shown in FIGURE 10a. It has three distinct tracks, as shown on the unfolded cylinder. The size of the small circles represents the pulse height, or photoelectron count, of the lit phototubes. The event also has a possible (but not definite) delayed pulse from a $\mu \rightarrow e$ decay (shown in inset). The event can be interpreted as a muon (weakest track) plus two showers arising from $K^{\circ} \rightarrow 2 \pi^{\circ}, \eta^{\circ} \rightarrow 2 \gamma$, or simply two nonresonant gammas or electrons. The event is nearly planar, having $\Sigma \theta_{i j}=340^{\circ}$. The background from atmospheric neutrinos is 
estimated as $\leq 0.3$ events at $90 \%$ C.L. One problem in interpreting the event as a nucleon decay is the relatively high momentum imbalance. The two showering tracks have a net momentum of about $485 \mathrm{MeV} / c$, which when added to the momentum of the "muon" track, leaves $380 \mathrm{MeV} / c$ net momentum. This is rather large for Fermi momentum from the oxygen nucleus. One would need to see some similar events with lower net momentum in order to make a case for this one.

The second event (FIGURE 10b) is interpreted as possibly having five tracks. The minimum energy for the event is $600 \mathrm{MeV}$. There is no evidence of $\mu \rightarrow e$ decay. Of course, a neutrino interaction having five primary tracks at this energy would be very unusual. On the other hand, charged pions are very likely to scatter in water at these

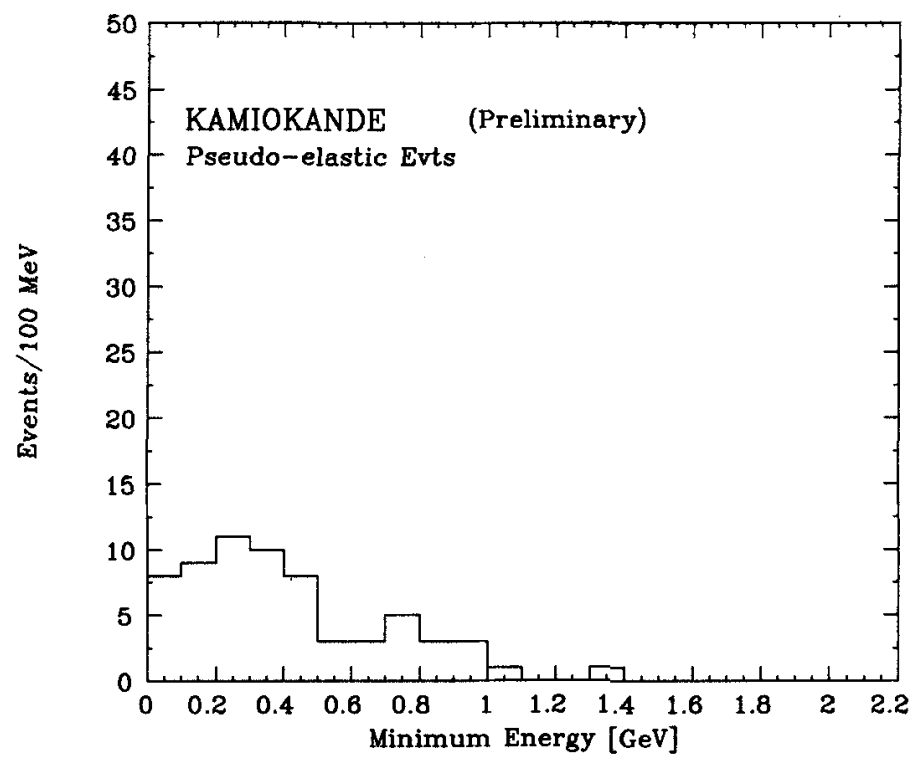

FIGURE 9. Energy distribution for 65 KAMIOKANDE contained events that appear to be pseudo-elastic neutrino interactions. Note that this detector can record events below $100 \mathrm{MeV}$.

energies so that one track can appear as two. The estimated neutrino background for this topology is $\leq 0.8$ events at $90 \%$ C.L. If the Kamioka group interprets the event as $\mathrm{p} \rightarrow e^{+} \omega^{\circ}\left(\omega^{\circ} \rightarrow \pi^{+} \pi^{-} \pi^{\circ}\right)$, it gets a proton mass of $880 \mathrm{MeV}$ (consistent with 940 within errors) and a net momentum of $250 \mathrm{MeV} / c$.

The third KAMIOKANDE candidate (FIGURE 10c) is a wide-angle $\left(\theta=166^{\circ}\right)$ two-track event with an energetic $(535 \mathrm{MeV} / c)$ showering track and a low momentum muon or pion of about $200 \mathrm{MeV} / c$. The invariant mass of the pair is about $720 \mathrm{MeV}$ with a momentum imbalance of more than $300 \mathrm{MeV} / \mathrm{c}$. In order to fit a nucleon decay mode, one needs to assume a third particle below Cherenkov threshold, thus making decays such as $N \rightarrow \mu^{+} \rho^{-}$or $\mathrm{p} \rightarrow \nu \rho^{+}$possible. The event has a definite $\mu \rightarrow e$ decay. 


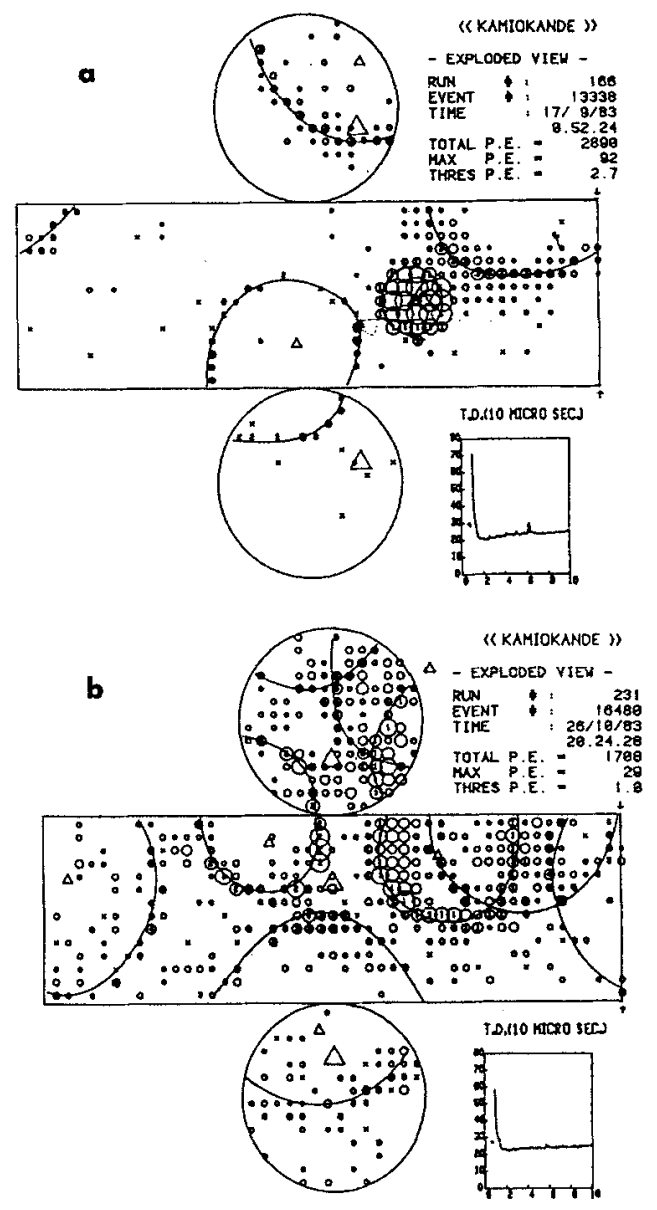

FIGURE 10a,b. KAMIOKANDE nucleon decay candidates. The small circles represent PMT hits projected onto an unfolded cylinder with the size proportional to light level. The large circles are drawn by the experimenters to guide the eye. Inset at lower right shows summed light output for the whole detector and indicates the presence or absence of stopping muon decays.

The fourth candidate (FIGURE 10d) also has a $\mu \rightarrow e$ decay and is a wide-angle $\left(146^{\circ}\right)$ two-track event with both tracks being nonshowering. The tracks have 404 and 203 photoelectrons, respectively. Assuming a $\pi \mu$ pair, one gets an invariant mass of 650 $\mathrm{MeV}$ and a net momentum of $230 \mathrm{MeV} / c$. Hence, it is also necessary in this case to invoke an unseen particle in order to fit nucleon decay. Then modes such as $\mathrm{p} \rightarrow \mu^{+} \rho^{\circ}$, $\mu^{+} k^{\circ}$, or $N \rightarrow \nu \rho^{\circ}$ are possible. The background is calculated as $\leq 0.3$ events at $90 \%$ C.L. 


\section{IMB RESULTS}

\section{Collaborating Institutions:}

University of California Irvine, University of Michigan, Brookhaven National Laboratory, California Institute of Technology, Cleveland State University, Fermilab, University of Hawaii, Lawrence Livermore Laboratory, University College, Warsaw University, University of Illinois

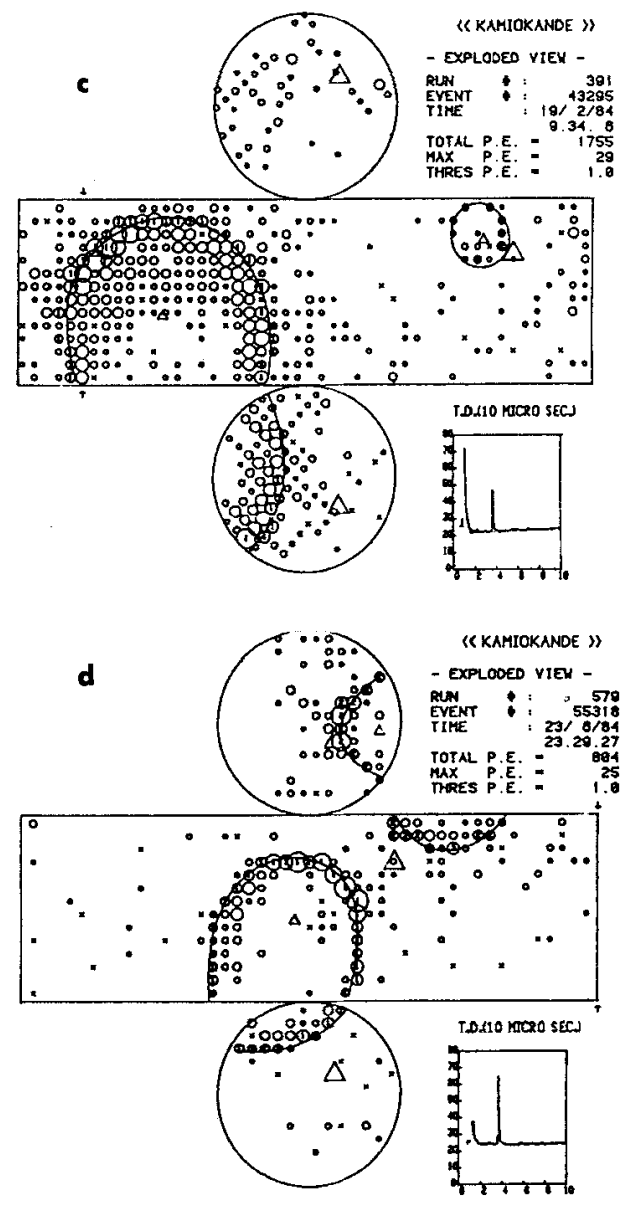

FIGURE 10c,d. KAMIOKANDE nucleon decay candidates. The small circles represent PMT hits projected onto an unfolded cylinder with the size proportional to light level. The large circles are drawn by the experimenters to guide the eye. Inset at lower right shows summed light output for the whole detector and indicates the presence or absence of stopping muon decays. 
Detector Characteristics:

Fiducial mass $=3300$ tonnes

Water Cherenkov type

20485 -inch hemispherical phototubes on the surfaces of a parallelepiped

$1.5 \%$ area coverage

Depth $=1670$ mwe

Results:

3800 tonne-yr exposure

401 contained events

21 nucleon decay candidates
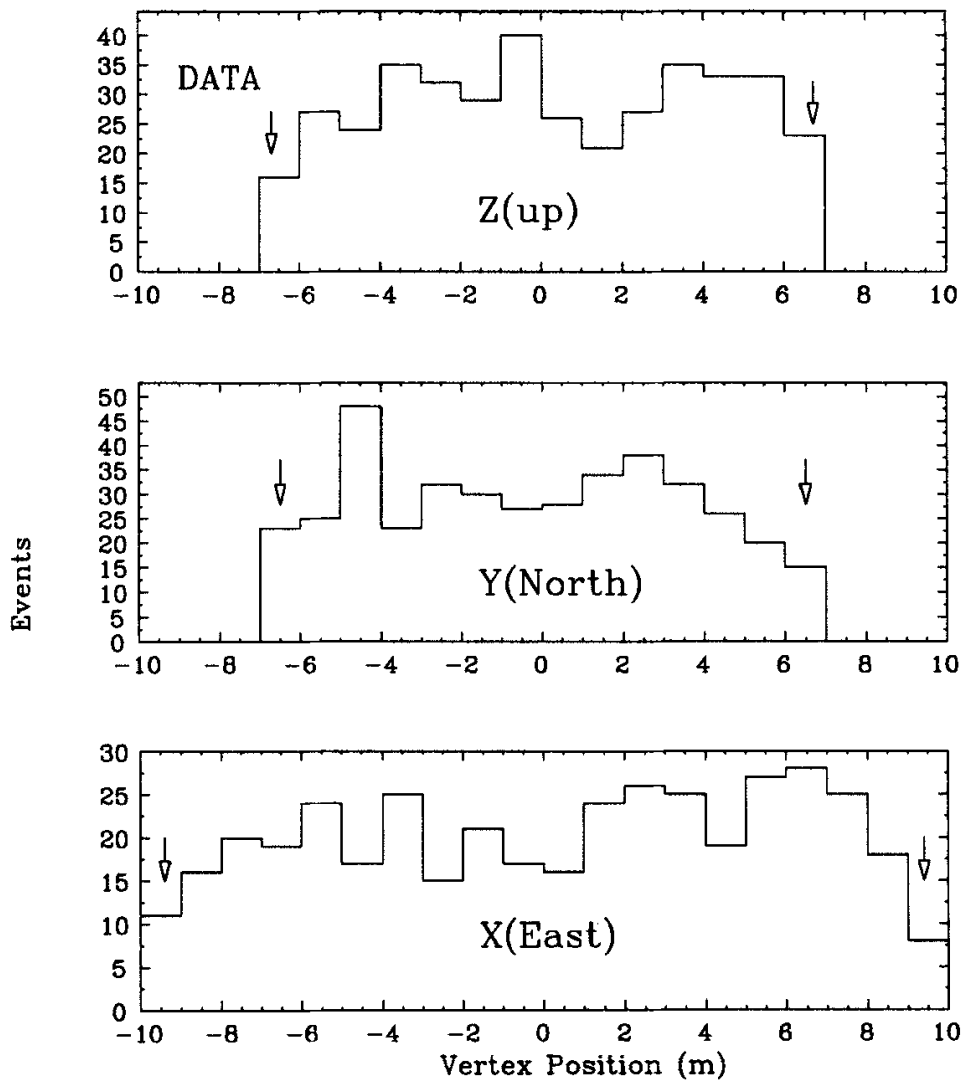

FIGURE 11. Projections of the vertices found in the IMB detector for 401 contained events. Arrows indicate the edges of the fiducial volume. 


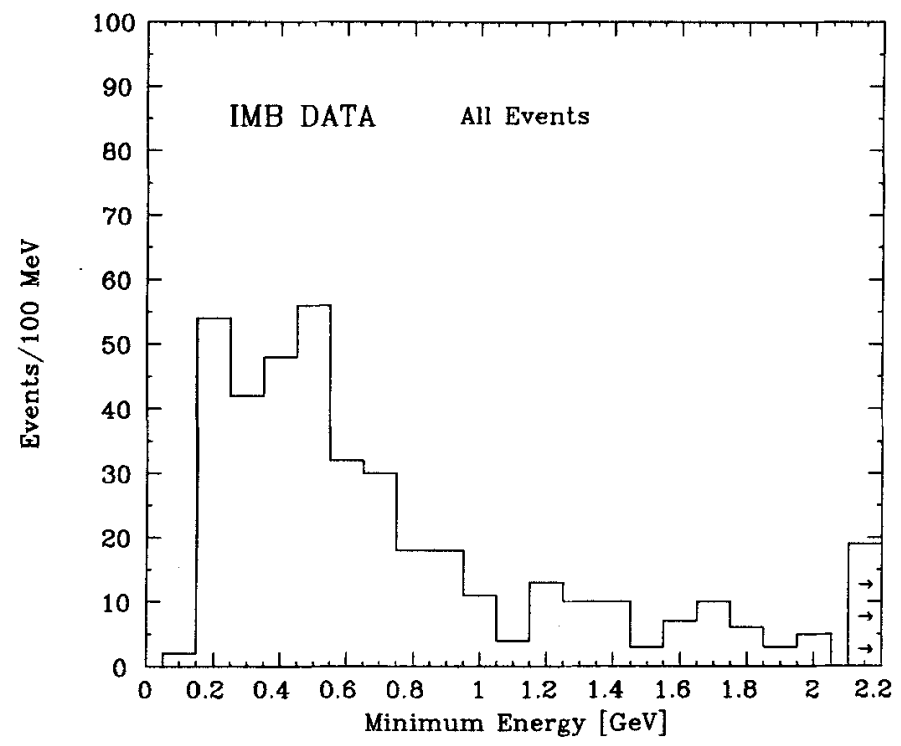

FIGURE 12. IMB energy distribution for 401 contained events.

The IMB collaboration has analyzed 417 live-days of data taken prior to the installation of the new wave-shifters around the tubes in the fall of 1984. The 401 contained events are distributed uniformly throughout the fiducial volume, which begins two meters in from the planes of tubes. FIGURE 11 shows the projections of the event vertices on the three orthogonal axes. Typical vertex resolutions are $\sim 90 \mathrm{~cm}$ for single-track, or narrow-angle, events that produce light mainly in one hemisphere and $\sim 50 \mathrm{~cm}$ for wide-angle events that have significant light recorded in two hemispheres.

The energy distribution for the 401 event sample is shown in FIGURE 12. Plotted here is the minimum visible energy $\left(E_{\min }\right)$. For events with all showering tracks, this is the total equivalent Cherenkov energy, $E_{c}$. When there is a muon decay detected, indicating the presence of a charged pion or muon, we define:

$$
E_{\min }=E_{c}+(200 \mathrm{MeV} \text { for each observed muon }) \text {. }
$$

The expected shape of the $E_{\min }$ distribution is shown simulated in FIGURE 13. The simulation is done by using neutrino and antineutrino events from the Gargamelle (Freon) bubble chamber data. ${ }^{6}$ Only charged-current events with a final-state muon are used. The muon is replaced by an electron to simulate $\nu_{e}$ events and by a neutrino to simulate neutral currents. The events are chosen proportionate to their occurrence in the atmospheric spectrum. They are then used to generate simulated events in the detector. The simulated events are passed through the same analysis programs as are the real data. The absolute rates agree to within $\pm 20 \%$ with estimates based on recent atmospheric flux calculations. ${ }^{2,4}$ 
In Figure 12, there is no evidence for a peak near the rest mass $(940 \mathrm{MeV})$ of the nucleon. One can make use of event geometry in order to dig further into the neutrino background. Neutrino events tend to be single-track (or narrow-angle) two-track events at these energies, whereas nucleon decays should be wide-angle events with opening angles in the range of $150^{\circ}-180^{\circ}$. We make use of an "anisotropy" variable $(A)$ to differentiate between the two types of events. The value of $A$ lies between 0 and 1 and is given by the mean vector sum of the unit vectors from the event vertex to each recorded phototube (PMT) hit. For a single short track that emits light only at the Cherenkov angle, $A$ will be given by the cosine of the angle ( 0.75 for water).

A scatterplot of $E_{\min }$ versus $A$ is shown in Figure 14. The region below and to the left of the dashed line is of interest for searching for nucleon decay. (The squares are events with two muon decays. Those and the three circled events will be discussed later.) The lower limit of $180 \mathrm{MeV}$ in FIGURE 14 is due to the software threshold cut of 40 PMTs. One notices that the population of the regions below $1000 \mathrm{MeV}$ are reasonable extrapolations of the regions above $1000 \mathrm{MeV}$ for both the "isotropic" $(A<0.5)$ data and the "anisotropic" $(A>0.5)$ data. Hence, even without external knowledge of the neutrino background, one can judge from these extrapolations that there is a sizable neutrino background that must be dealt with in searching for nucleon decay, even in the "wide-angle" event sample.

If one uses just the two variables, $E_{\min }$ and $A$, to describe the events, then most of the events inside the dashed area of FIGURE 14 are "candidates" for one or more of the nucleon decays for which limits have recently been published. ${ }^{7}$ (These published limits

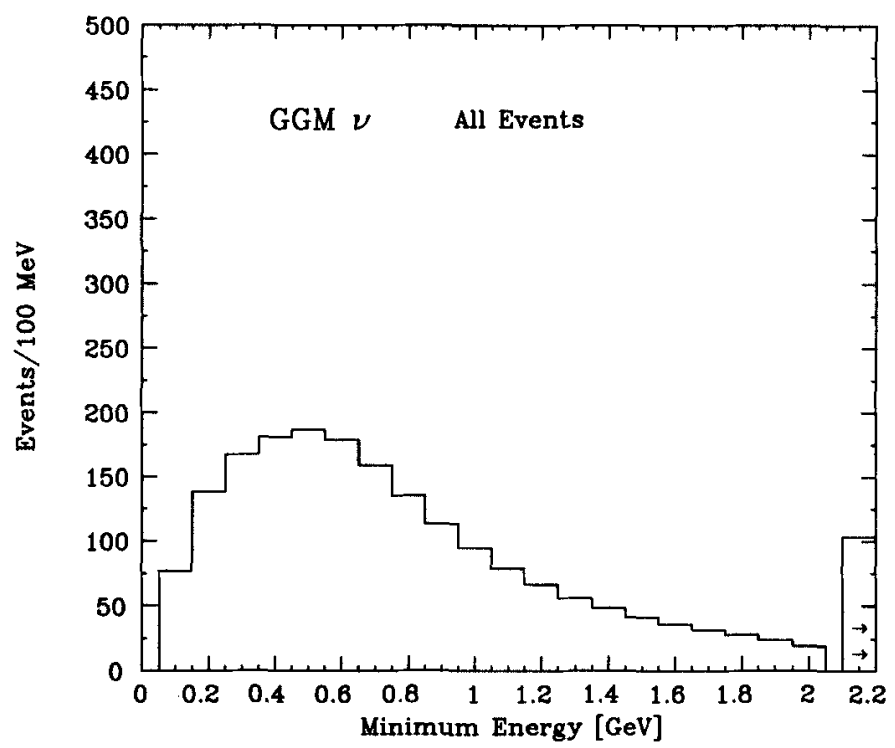

FIGURE 13. Energy distribution for IMB simulated events based on Gargamelle bubble chamber data. This represents the shape of the atmospheric neutrino background. 


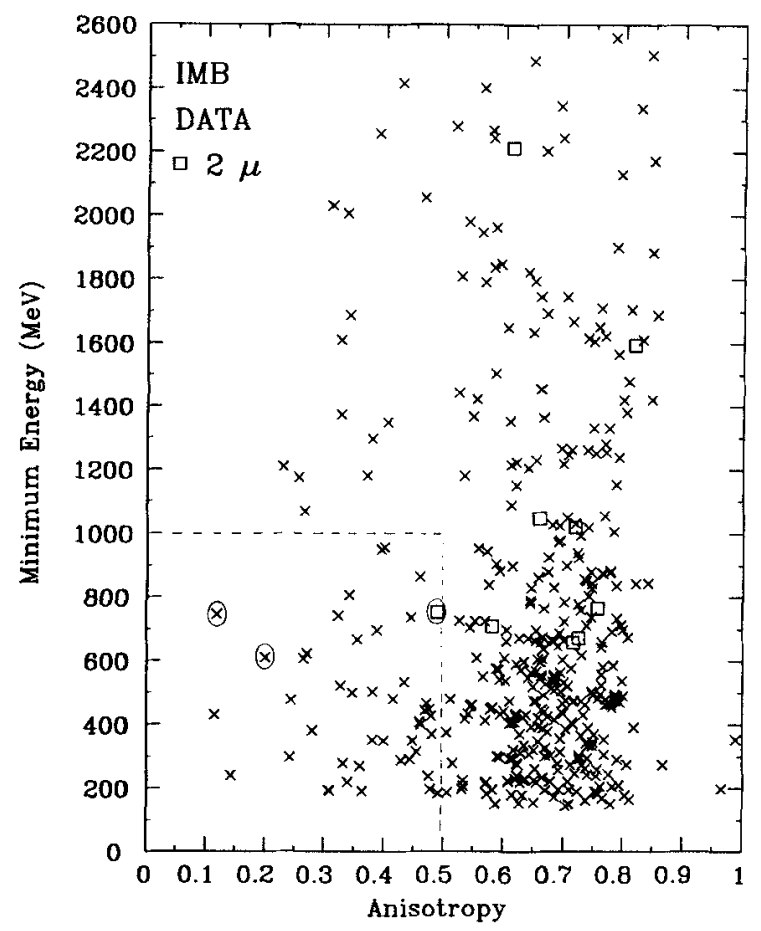

FIGURE 14. Scatterplot of $E_{\min }$ versus $A$ for 401 IMB contained events. Open squares are events with two muon decays. (There is one additional $2 \mu$ event at $2700 \mathrm{MeV}, A=0.46$.) Circled events are shown in Figures 15a, c, and e.

are conservative estimates calculated by taking the neutrino background to be negligible.)

In general, there is much more information in each event than just $E_{\min }$ and $A$. We have selected three particularly interesting events (circled on FIGURE 14) for closer inspection. The first event is shown in FIGURE 15a. Each lit PMT is indicated by one or more slashes, with each slash being proportional to the amount of light it recorded. The PMT hits have been projected onto a sphere whose center is at the event vertex. The sphere has been unfolded into a standard Mercator projection with the polar axis perpendicular to the track directions. The event is a nice example of a wide-angle two-track event, as indicated by two well-separated Cherenkov rings. The angle between the two tracks is measured as $178^{\circ}$ with a typical error of $\sim 10^{\circ}$.

There are two recorded muon decays (not shown) in the event. It is the only one of ten such events (see FIGURE 14) that is similar to the HPW events discussed earlier.

The left track in Figure $15 \mathrm{a}$ has $E_{c}=301 \mathrm{MeV}$ and the right track has $E_{c}=68$ $\mathrm{MeV}$. [Typical errors in $E_{c}$ are $\pm 100 \% / \sqrt{ }$ PMTs $\pm 10 \%$ (sys.).] The event is a candidate for the following decay modes:

$$
N \rightarrow \mu^{-} \pi^{+}
$$




$$
\begin{aligned}
& P \rightarrow \mu^{+} \mu^{+} \mu^{-} \\
& P \rightarrow \mu^{+}+\left(K^{\circ}, \eta^{\circ}, \rho^{\circ}, \omega^{\circ}\right) .
\end{aligned}
$$

Taking the first interpretation, $N \rightarrow \mu^{-} \pi^{+}$, for example, one derives the following kinematics:

$$
\begin{aligned}
P_{\mu} & =490 \mathrm{MeV} / c \\
P_{\pi} & =297 \mathrm{MeV} / c \\
\Delta P & =193 \mathrm{MeV} / c \\
M & =806 \pm 120 \mathrm{MeV} .
\end{aligned}
$$
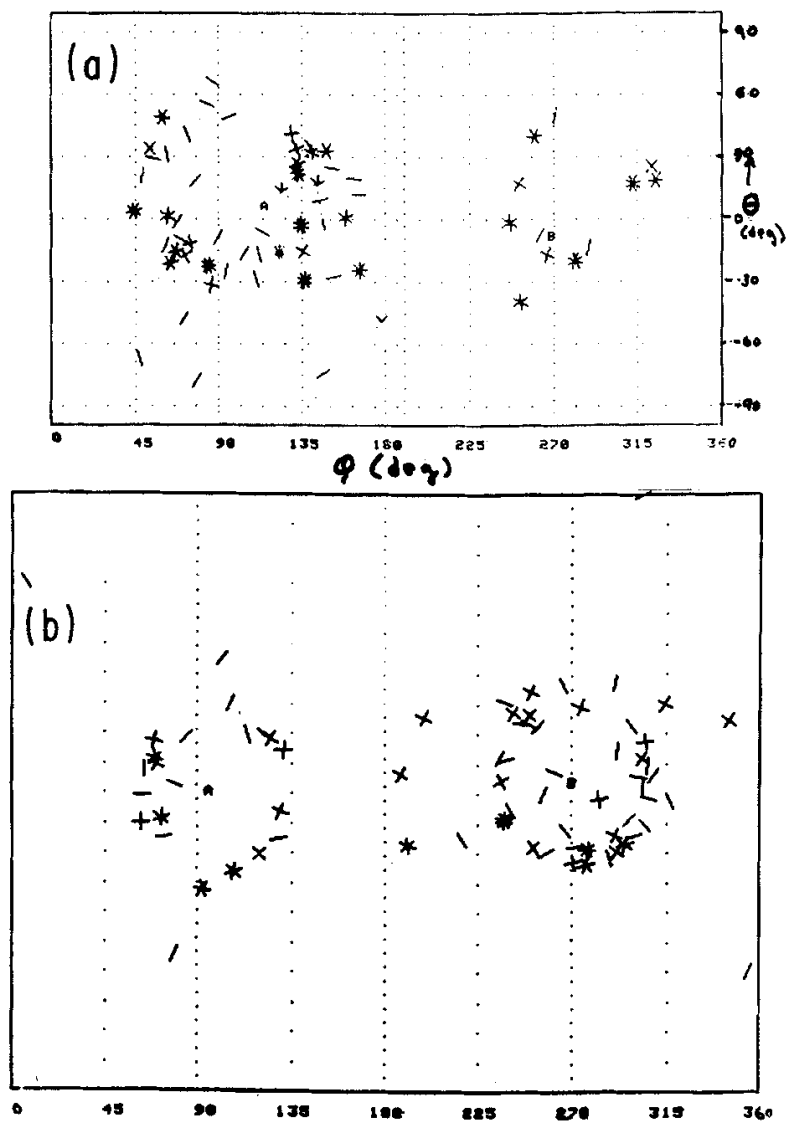

FIGURE 15a,b. (a) Wide-angle two-track events from the IMB detector (Event $8320 ; 2 \mu$ ). (b) Simulated wide-angle two-track events from neutrino interactions $\left(\nu N \rightarrow \mu^{-} \Delta^{+} \rightarrow \mu^{-} \pi^{+} N\right)$. The points labeled " $A$ " and " $B$ " represent the reconstructed track directions as seen from the vertex. (See text.) 

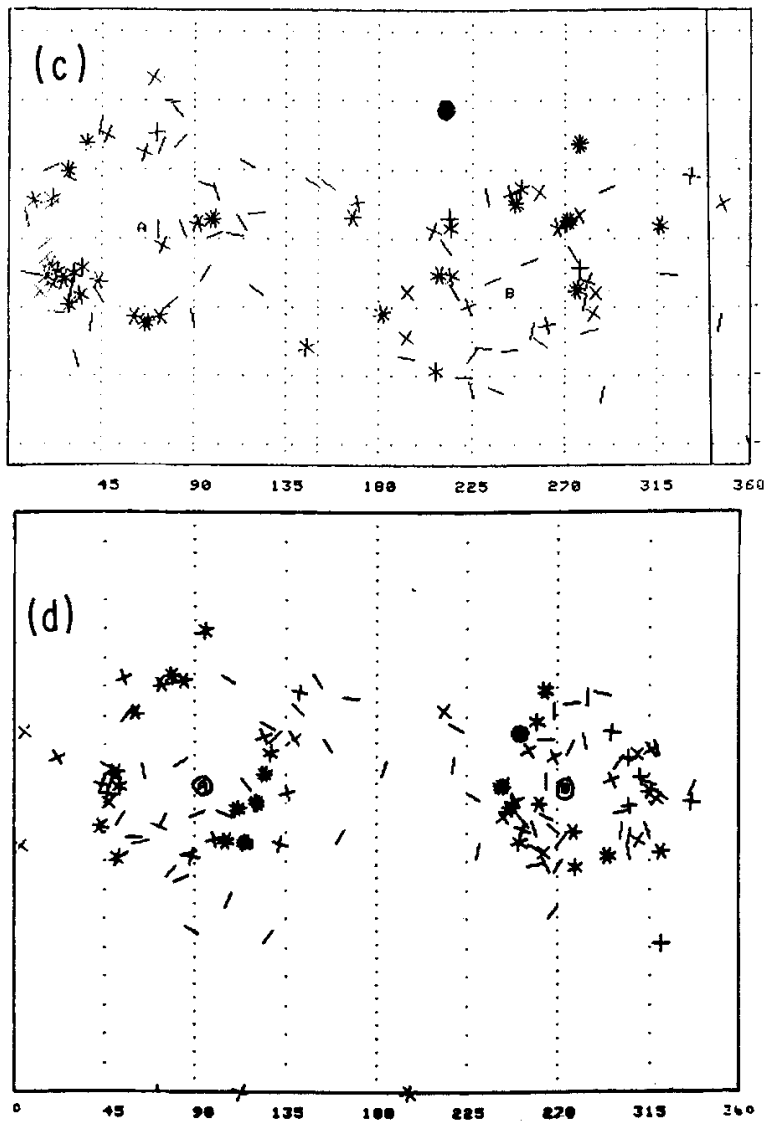

FIGURE 15c,d. (c) Wide-angle two-track events from the IMB detector (Event 17, 473; no $\mu$ ). (d) Simulated wide-angle two-track events from neutrino interactions $\left(\nu N \rightarrow e^{-} \pi^{+} N\right)$. The points labeled " $A$ " and " $B$ " represent the reconstructed track directions as seen from the vertex. (See text.)

Thus, although the invariant mass is somewhat low, it gives a reasonable fit to bound neutron decay.

Now, what about the background?

As mentioned earlier (FIGURE 2), atmospheric neutrinos do produce wide-angle two-track events. Figure 15b shows a simulated background event that has been passed through the standard analysis programs. It is quite similar to the real event of FIgURE 15a. The included angle is $174^{\circ}$. The background is $\nu N \rightarrow N \mu^{-} \pi^{+}$. We will estimate the frequency of such background events later in the discussion.

The second interesting real event is shown in FIGURE 15c. It has no recorded muon decays, but the total Cherenkov energy is $608 \mathrm{MeV}$, as opposed to $369 \mathrm{MeV}$ for the first event. Its included angle is $\sim 175^{\circ}$ and it is a candidate for several nucleon decay modes, including $N \rightarrow e^{+} \pi^{-}, e^{+} \rho^{-}, e^{-} \rho^{+}$. The background calculation also, in this case, 
has a similar event, which is shown in FIGURE 15d. The background reaction is $\nu N \rightarrow$ $\mathrm{Ne}^{-} \boldsymbol{\pi}^{+}$.

The third wide-angle event of interest is shown in Figure 15e. Its Cherenkov energy is still higher: $E_{c}=722 \mathrm{MeV}$. It also has no recorded muon decays and is a candidate for the same modes as the second event. It falls just below the acceptance cut
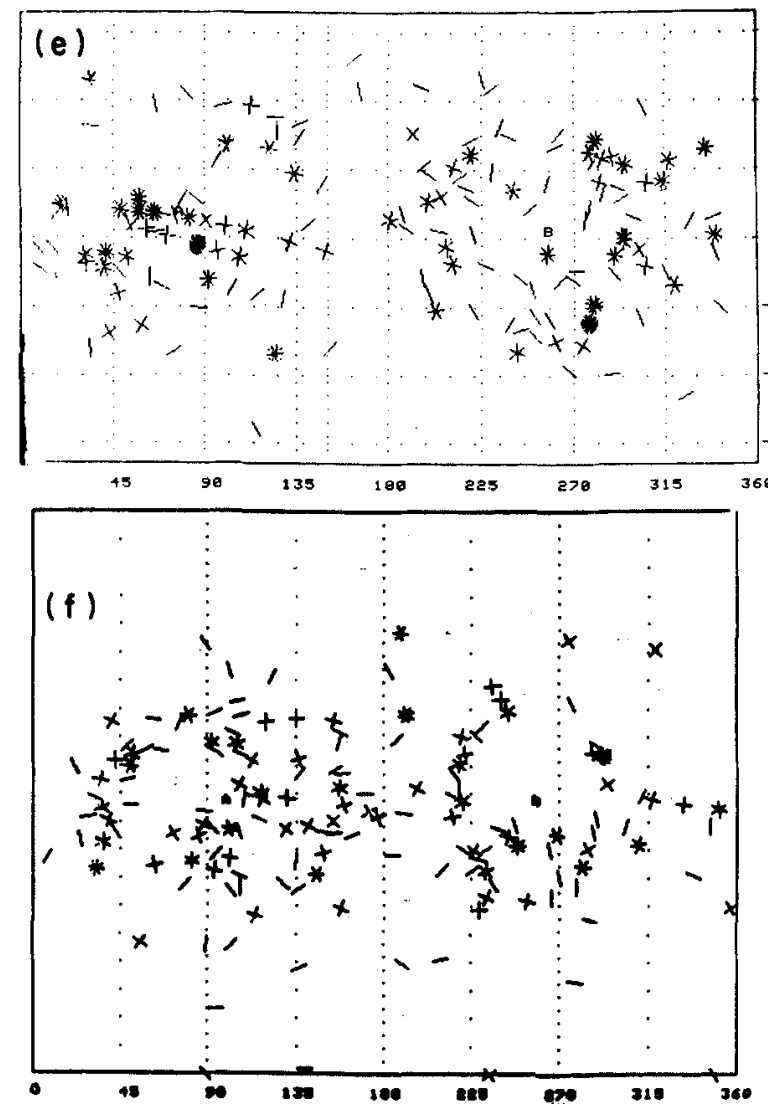

FIGURE 15e,f. (e) Wide-angle two-track events from the IMB detector (Event 42,652; no $\mu$ ). (f) Simulated wide-angle two-track events from neutrino interactions $\left(\nu N \rightarrow e^{-} \pi^{+} N\right)$. The points labeled " $A$ " and " $B$ " represent the reconstructed track directions as seen from the vertex. (See text.)

$\left(E_{c}=750 \mathrm{MeV}\right)$ for $\mathrm{p} \rightarrow e^{+} \pi^{\circ}$. It also has a companion simulated background event, shown in FIGURE 15f, from the reaction of $\nu \rightarrow N e^{-} \pi^{\circ}$.

We see that wide-angle $\left(>150^{\circ}\right)$ two-track events consistent with nucleon decay definitely are present in the IMB data at a level of $\sim 1 \%$ of the contained events. The question is, of course, are they nucleon decay? What is the expected background rate? 
The cross sections for single pion production by neutrinos in the energy range of 1-3 GeV are fairly well known. I make the following estimate of the rate for wide-angle two-track events to pass the IMB selection criteria, using atmospheric neutrinos to generate single pions in charged-current interactions.

I expect 1.1 events/kilotonne-yr having the following properties:

(1) $0.2<E_{c}<1.0 \mathrm{GeV}$;

(2) Two tracks both with $E_{c}>50 \mathrm{MeV}$;

(3) Included angle $>150^{\circ}$.

This estimate is in good agreement, given the statistics of a few events, with the rate seen by IMB.

\section{CONCLUSIONS}

(1) Atmospheric neutrino interactions have been recorded in several of the "modern" nucleon decay detectors. The rate of detected events is about as expected:

$$
\sim 100 \text { events/kt-yr with } E_{\text {vig }}>0.2 \mathrm{GeV} \text {. }
$$

(2) There are approximately five events/kt-yr that have $\geq$ two fairly wide-angle tracks and that fall into the general category of nucleon decay "candidates."

(3) Wide-angle $\left(>150^{\circ}\right)$ two-track events with $\Delta P \approx 200 \mathrm{MeV} / c$ (the "classic" proton decay topology) do occur at a rate of $\sim$ one event/kt-yr. At this point, there is no good evidence that such events are inconsistent with the background; the rate is approximately as expected.

(4) Using the above numbers and adding some reasonable estimate of the background to be subtracted, one gets the following general limits:

$$
\begin{aligned}
& \text { If } P \rightarrow \text { "classic" modes, then } \tau_{p} \geq 5 \times 10^{32} \mathrm{yr} \text {; } \\
& \text { If } P \rightarrow \nu \text { dominated modes, then } \tau_{p} \geq 5 \times 10^{31} \mathrm{yr} \text {. }
\end{aligned}
$$

(5) If nucleon decays are hiding in the "neutrino curtain," then there is going to be a lot of hard work digging them out. One helpful clue should be the dominance of a single decay mode.

\section{THE SCHEDULED FUTURE}

We return to the fact that the existence of proton decay is a very important question and one that hopefully can be answered experimentally. The following experiments are being mounted to work on the question:

(1) The KFG group is building a new 300-tonne detector with better spatial resolution ( $6 \mathrm{~mm}$ plates). It will be ready in the summer of 1985 .

(2) The Frejus detector will be increased to 900 tonnes in the spring of 1985.

(3) The Kamioka detector will be surrounded with a water Cherenkov anti- 
counter to increase the fiducial volume and lower the energy threshold. Timing information will be added to the tubes to improve spatial resolution.

(4) The IMB group has increased the light collection of its detector by a factor of two and will add another factor of two by the end of 1985 .

(5) The Soudan-2 group (Minnesota, Argonne, Oxford, Rutherford, Tufts) will build a new detector with excellent $(2 \mathrm{~mm})$ spatial resolution and good ionization information. The group plans to have 500 tonnes operating in 1986 and 1000 tonnes in 1987.

Clearly the subject is alive and well; the question remains, is the proton? Time and patience, hopefully, will tell.

In closing, let me say that I'm very impressed with the way Martin Block and his cohorts have put together this conference. The physics, the hospitality, the recreation (including the fine dinners), and the camaraderie have all been first-rate. It's hard for a physicist not to find something wrong with an experiment, but this one has been nearly perfect; so good that it needs to be repeated.

\section{ACKNOWLEDGMENTS}

I am grateful to D. Ayres, R. Barloutaud, D. Cline, E. Fiorini, M. Marshak, V.S. Narasimham, and D. Perkins for providing much of the information on which this paper is based. Special thanks go to my colleagues on the IMB experiment (see reference 7) for their untiring efforts in producing and analyzing the data presented here, much of which is as yet unpublished.

\section{NOTES AND REFERENCES}

1. Perkins, D. H. Proton decay experiments. To appear in Ann. Rev.

2. Gaisser, T. K. \& T. STANEv. 1984. In Proceedings of the International Colloquium on Baryon Nonconservation, Salt Lake City, January, 1984. D. Cline, Ed.: 61. University of Wisconsin Press. Madison, Wisconsin; Gaisser, T. K. 1984. In Proceedings of the Eleventh International Conference on Neutrino and Astro Physics. K. Kleinknecht \& E. A. Paschos, Eds.: 372. World Scientific. Singapore.

3. Figures 7 and 8 and TABle 2 are taken from the Ph.D. Thesis of More, A. H. 1984. University of Wisconsin.

4. Perkins, D. H. 1984. Atmospheric neutrino fluxes. Oxford University preprint 85/84.

5. Koshiba, M. 1984. Plenary Report, XXII International Conference on High Energy Physics, Leipzig. See FIGURE 15 of this report for a comparison of $\tau / B$ limits from various experiments. IMB limits since then have been updated in reference 7.

6. DedEN, H. et al. 1975. Nucl. Phys. B85: 269. We are grateful to the Gargamelle Collaboration for supplying us with summary tapes of their data.

7. Park, H. S., G. BlewitT et al. 1985. Phys. Rev. Lett. 54: 22. An updated table of IMB lifetime limits based on twice the livetime cited here will be available in the spring of 1985. 